\title{
Article \\ Sensitivity to Cisplatin in Head and Neck Cancer Cells Is Significantly Affected by Patient-Derived Cancer-Associated Fibroblasts
}

\author{
Barbora Peltanova ${ }^{1}$ (D), Marketa Liskova ${ }^{1}$, Jaromir Gumulec ${ }^{1}\left(\mathbb{D}\right.$, Martina Raudenska ${ }^{1,2,3}$, \\ Hana Holcova Polanska ${ }^{1,2}$, Tomas Vaculovic ${ }^{4}$, David Kalfert ${ }^{5}$ (D), Marek Grega ${ }^{6}$, Jan Plzak ${ }^{5}$, Jan Betka ${ }^{5}$ and \\ Michal Masarik 1,2,7,*
}

\section{check for} updates

Citation: Peltanova, B.; Liskova, M.; Gumulec, J.; Raudenska, M.;

Polanska, H.H.; Vaculovic, T.; Kalfert,

D.; Grega, M.; Plzak, J.; Betka, J.; et al. Sensitivity to Cisplatin in Head and Neck Cancer Cells Is Significantly Affected by Patient-Derived Cancer-Associated Fibroblasts. Int. J. Mol. Sci. 2021, 22, 1912. https:// doi.org/10.3390/ijms22041912

Academic Editor: A. Phillip West

Received: 6 January 2021

Accepted: 6 February 2021

Published: 15 February 2021

Publisher's Note: MDPI stays neutral with regard to jurisdictional claims in published maps and institutional affiliations.

Copyright: (C) 2021 by the authors Licensee MDPI, Basel, Switzerland. This article is an open access article distributed under the terms and conditions of the Creative Commons Attribution (CC BY) license (https:// creativecommons.org/licenses/by/ $4.0 /)$.
1 Department of Pathological Physiology, Faculty of Medicine, Masaryk University, Kamenice 5 CZ-625 00 Brno, Czech Republic; bpeltanova@seznam.cz (B.P.); liskovam@tiscali.cz (M.L.); j.gumulec@med.muni.cz (J.G.); m.raudenska@gmail.com (M.R.); hana.polanska@gmail.com (H.H.P.)

2 Department of Physiology, Faculty of Medicine, Masaryk University, Kamenice 5, CZ-625 00 Brno, Czech Republic

3 Department of Chemistry and Biochemistry, Mendel University, Zemedelska 1, CZ-613 00 Brno, Czech Republic

4 Department of Chemistry, Faculty of Science, Masaryk University, Kotlarska 2, CZ-611 37 Brno, Czech Republic; vaca_777@yahoo.com

5 Department of Otorhinolaryngology and Head and Neck Surgery, University Hospital Motol, First Faculty of Medicine, Charles University, V Uvalu 84, 15006 Prague 5, Czech Republic; david.kalfert@fnmotol.cz (D.K.); Jan.Plzak@fnmotol.cz (J.P.); jan.betka@fnmotol.cz (J.B.)

6 Department of Pathology and Molecular Medicine, 2nd Faculty of Medicine, Charles University and University Hospital Motol, V Uvalu 84, 15006 Prague 5, Czech Republic; marek.grega@fnmotol.cz 7 BIOCEV, First Faculty of Medicine, Charles University, Prumyslova 595, CZ-252 50 Vestec, Czech Republic

* Correspondence: masarik@med.muni.cz; Tel.: +420-5-4949-3631

Abstract: Cancer-associated fibroblasts (CAFs) are one of the most abundant and critical components of the tumor stroma. CAFs can impact many important steps of cancerogenesis and may also influence treatment resistance. Some of these effects need the direct contact of CAFs and cancer cells, while some involve paracrine signals. In this study, we investigated the ability of head and neck squamous cell carcinomas (HNSCC) patient-derived CAFs to promote or inhibit the colony-forming ability of HNSCC cells. The effect of cisplatin on this promoting or inhibiting influence was also studied. The subsequent analysis focused on changes in the expression of genes associated with cancer progression. We found that cisplatin response in model HNSCC cancer cells was modified by coculture with CAFs, was CAF-specific, and different patient-derived CAFs had a different "sensitizing ratio". Increased expression of VEGFA, PGE2S, COX2, EGFR, and NANOG in cancer cells was characteristic for the increase of resistance. On the other hand, CCL2 expression was associated with sensitizing effect. Significantly higher amounts of cisplatin were found in CAFs derived from patients who subsequently experienced a recurrence. In conclusion, our results showed that CAFs could promote and/or inhibit colony-forming capability and cisplatin resistance in HNSCC cells via paracrine effects and subsequent changes in gene expression of cancer-associated genes in cancer cells.

Keywords: head and neck cancer; cancer-associated fibroblasts; cisplatin; treatment resistance; cancer recurrence; patient-derived cell cultures; coculture

\section{Introduction}

Head and neck squamous cell carcinomas (HNSCC) are the sixth most common cancer worldwide. HNSCC tumors emerge in the upper aero-digestive tract (including the oral cavity, pharynx, and larynx). Surgery and radiotherapy are effective in the treatment of early-stage tumors (stage I and II). Nevertheless, about $70 \%$ of patients are diagnosed with 
an advanced stage III or IV. The locoregional disease recurs in $60 \%$ of patients and metastatic disease arises in 15-25\% [1]. For these reasons, chemotherapy (alone or in combination with radiotherapy) has an important role in the treatment of HNSCC. Currently, the key anticancer drug for advanced HNSCC is still cisplatin (cis-diamminedichloroplatinum; CDDP) [2,3]. Nevertheless, HNSCC represents a heterogeneous group of tumors with varying levels of cisplatin resistance. CDDP administration in resistant cases would provide almost no curative effect but could increase the chance of adverse side effects and disease progression. Recently, it was shown that stromal cells in tumor tissue may modulate the resistance to chemotherapy [4]. Moreover, it was shown that drug resistance is likely to be reversible by removal of the tumor-supporting stromal cells [5]. The effect of the tumor microenvironment (TME) on resistance to therapy is easy to understand since different soluble factors produced by the TME can trigger pro-survival pathways in tumor cells, especially when damaged by chemotherapy $[6,7]$. The most represented cells within the stroma are cancer-associated fibroblasts (CAFs).

In this study, we investigated the ability of HNSCC patient-derived CAFs to promote or inhibit the colony-forming capability of HNSCC cells, and the effect of cisplatin on this promoting or inhibiting influence. Subsequent analysis on FaDu and Detroit562 cell lines focused on changes in the expression of genes associated with: (1) pluripotency (NANOG, SOX2, POU5F, SNAIL); (2) cell division (FOLR1); (3) proliferative activity of tumor cells (MKI67); (4) angiogenesis (VEGFA); (5) acquisition of autonomous proliferative signalling (EGFR, EGF); (6) immune response (COX2, CCL2, IL6, EP3, PGE2S); and (7) cell cycle, and cell death modifications (BCL2, BIRC5, NFKB1, CAV1).

\section{Results}

\subsection{Clinical Characterization of Patients and Tumors Used for CAFs Preparation}

Primary cultures were created from seven head and neck squamous cell primary tumors. Of those, one sample was from a female and three were p16-positive. Tumor location was as follows: three laryngeal, three oropharyngeal, and one hypopharyngeal. Two patients exhibited recurrence after successful therapy. Patient characteristics are shown in Table 1.

Table 1. Clinico-pathological characterization of head and neck squamous cell carcinomas (HNSCC) patients based on TNM staging and grading (8th revision).

\begin{tabular}{|c|c|c|c|c|c|c|c|c|c|c|c|c|}
\hline Patient & Gender & $\begin{array}{c}\text { Age at } \\
\text { Diagnosis }\end{array}$ & $\begin{array}{l}\text { Tumor } \\
\text { Location }\end{array}$ & $\mathrm{pT}$ & $\mathrm{pN}$ & $\mathbf{c M}$ & G & p16 & Stage & $\begin{array}{c}\text { Surgery + } \\
\text { Adjuvant } \\
\text { Radiotherapy }\end{array}$ & $\begin{array}{c}\text { Adjuvant } \\
\text { Chemotherapy }\end{array}$ & Recurrence \\
\hline M5.1 & $\mathrm{F}$ & 59 & larynx & $4 a$ & $2 b$ & 0 & 3 & 0 & IVA & 1 & 0 & 0 \\
\hline M6.1 & M & 81 & oropharynx & 2 & 0 & 0 & 2 & 0 & $\mathrm{I}$ & 1 & 0 & 0 \\
\hline M7.1 & M & 60 & hypopharynx & 2 & $2 b$ & 0 & 2 & 1 & IVA & 1 & 0 & 1 \\
\hline M9.1 & M & 54 & oropharynx & 2 & 1 & 0 & 2 & 1 & $\mathrm{I}$ & 1 & 1 & 1 \\
\hline M11.1 & M & 63 & oropharynx & 2 & 2 & 0 & 3 & 1 & II & 1 & 1 & 0 \\
\hline M12.1 & M & 49 & larynx & $4 a$ & 1 & 0 & 3 & 0 & IVA & 1 & 0 & 0 \\
\hline M14.1 & M & 62 & larynx & $4 a$ & 0 & 0 & 2 & 0 & IVA & 1 & 0 & 0 \\
\hline
\end{tabular}

\subsection{Lineage Specificity of Patient-Derived CAFs and Preparation of Conditioned Media}

Patient-derived CAFs were prepared as early passage cell populations created by the overgrowth of the origin nal patient-derived primary culture from stromal fibroblasts presented in this primary culture. Population overgrowth by fibroblasts was successfully reached after two passages. The passages of CAFs used for further analyses ranged from one to four. The CAFs cell populations showed a typical spindle-shaped cell morphology (Figure 1a). Our selection method was confirmed by flow cytometry of three randomly selected early passage CAFs. The CD90 (a CAFs marker [8]) positivity of these selected cultures ranged between $95.2 \%$ and $97.2 \%$ (Figure 1b, Supplementary Figure S1). This indicated the successful establishment of the CD90+ CAF population. 
a

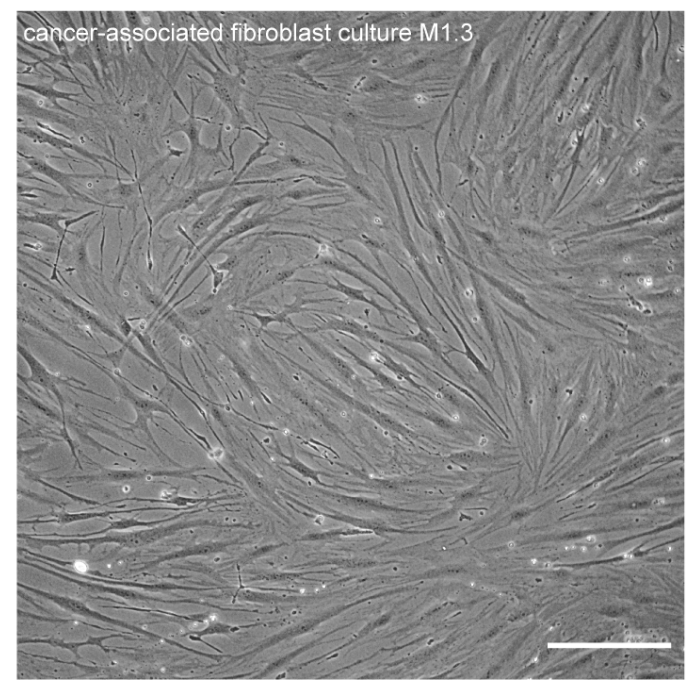

b
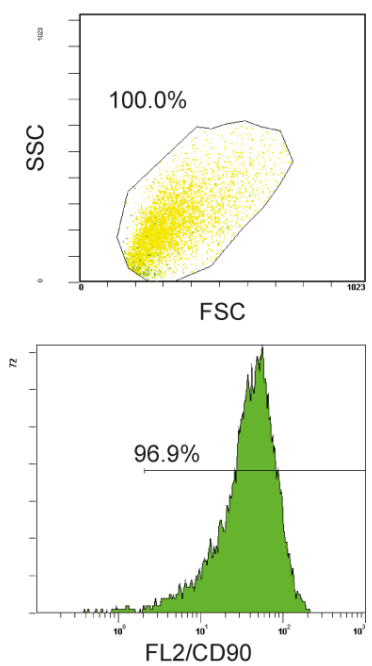

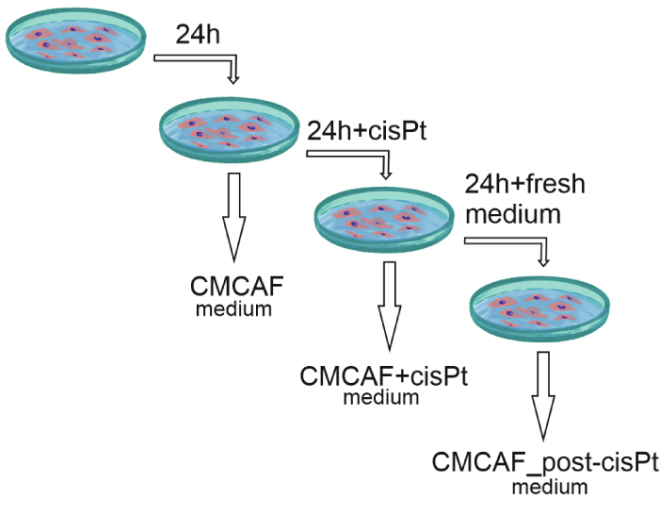

Figure 1. Preparation and characterization of cancer-associated fibroblasts (CAFs) and CAF-conditioned media. (a) Representative phase-contrast image of CAF culture, 20× magnification, scalebar indicates $100 \mu \mathrm{m}$. (b) Verification of fibroblast phenotype by CD90 status. Forward scatter and side scatter population (top) and FL2 histogram. (c) Scheme of CAF-derived conditioned media preparation for indirect coculture experiments. After the cells (CAFs) reached optimal confluence, their culture media were replaced with fresh media. After $24 \mathrm{~h}$ the conditioned media were collected (CMCAF) and replaced with media containing $5 \mu \mathrm{M}$ cisplatin. After $24 \mathrm{~h}$ the media were collected (CMCAF+cisplatin) and replaced with fresh media, which were collected after an additional $24 \mathrm{~h}$ (CMCAF_post-cisplatin).

After the cells (CAFs) reached optimal confluence, their culture media were replaced with fresh media. After $24 \mathrm{~h}$ the conditioned media were collected (CMCAF) and replaced with media containing $5 \mu \mathrm{M}$ cisplatin [9]. After $24 \mathrm{~h}$ the media were collected (CMCAF+cisplatin) and replaced with fresh media, which were collected after an additional $24 \mathrm{~h}$ (CMCAF_post-cisplatin) (see Figure 1c). These conditioned media were further used for gene expression experiments and platin amount assay.

\subsection{Patient-Derived CAFs Affect the Colony-Forming Capability of Cancer Cells}

To assess if CAF-derived compounds support head and neck cancer cell growth, CAFs were cocultured with FaDu cell line in a transwell system. CAFs derived from tumor tissue of individual HNSCC patients significantly differed in their ability to support or inhibit the colony-forming capability of FaDu cells (Figure 2a,b, Supplementary Table S1). Patient-derived early passage CAFs M.5.1 significantly inhibited the clonogenic survival of FaDu cells. On the other hand, patient-derived early passage CAFs M.6.1, M.7.1, and M.11.1 significantly supported the clonogenic capacity of FaDu cells. These were further named as tumor-supporting and tumor-inhibiting CAFs.

To test a hypothesis that CAFs may affect the sensitivity of cancer cells to cisplatin, CAF-FaDu coculture was exposed to $5 \mu \mathrm{M}$ cisplatin treatment. Transwell ${ }^{\circledR}$ cell culture inserts were used, which means the medium was shared between both cell populations (CAFs and cancer cells). Cisplatin was added to the cultivation medium and therefore influenced both cell populations. There was a systematic decrease in colony-forming capability after cisplatin treatment (Figure 2b,c), however, the extent of this decrease differed among CAFs and was expressed as a "sensitizing ratio" (Figure 2d; for calculation see the method section). Of those, CAFs M.5.1 and M6.1 were considered cisplatin-sensitizing. 


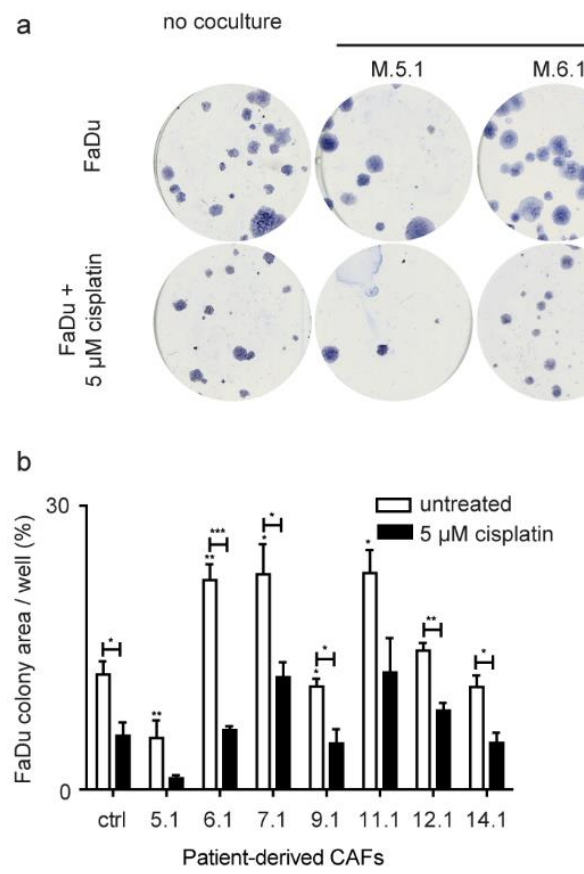

cocultured with patient-derived cancer-associated fibroblasts

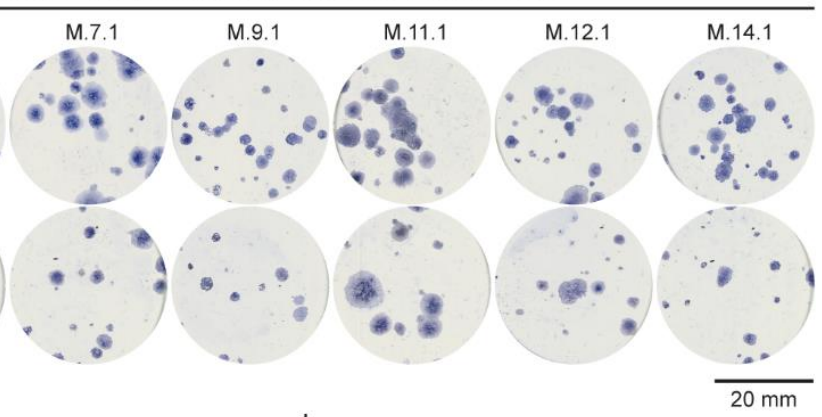

C

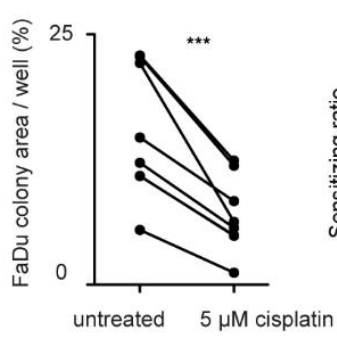

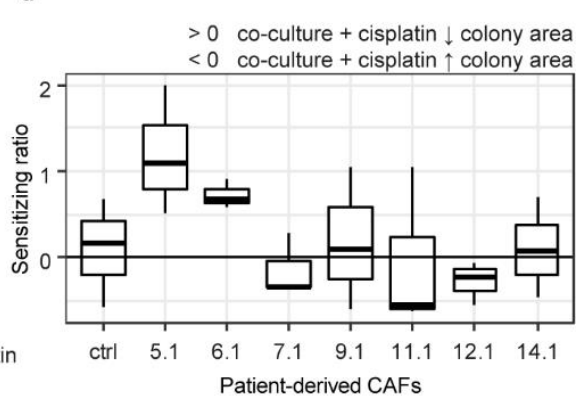

Figure 2. Effect of patient-derived CAFs on the area of cancer cell colonies. (a) Representative Coomassie-blue stained FaDu colonies in six-well plates. Patient-derived CAFs either promoted, inhibited, or had no effect on the formation of FaDu colonies, as shown by the different size of their area compared to control. (b) The total area of colonies relative to well area (percentage of area covered), not cocultured (ctrl) FaDu cells, and FaDu cocultured with CAFs (5.1-14.1). The asterisk above white bars shows a significant difference compared to not cocultured FaDu cells. Black bars showed coculture of FaDu cells with CAFs by the presence of cisplatin. Cisplatin showed significantly different efficacy in inhibiting the formation of the FaDu colonies after coculture with individual patient-derived CAFs. (c) Effect of cisplatin. Group comparison (paired test) between coculture with no treatment and coculture in presence of cisplatin showed a significant decrease in the total area of FaDu colonies in the treated group. $p$-values from group comparisons based on the t-test are shown. Asterisks represent statistical significance $\left.{ }^{*} p<0.05 ;{ }^{* *} p<0.01 ;{ }^{* * *} p<0.001\right)$. (d) The sensitizing ratio showing the extent of inhibition of colony-forming capacity after cisplatin treatment in cancer cells cocultured with patient-derived CAFs. Values above 0 indicate a higher level of inhibition when exposed to cisplatin. The control corresponded to sensitizing ratio of non-cocultured cells (equal to 0 ).

\subsection{The Colony-Forming Capability of FaDu Cells after CAF Coculture Is Related to Cancer-Associated Genes}

Gene expression analysis of FaDu cells was performed to link CAF colony-forming capability with respective signalling in cancer cells. VEGFA and NANOG expression was increased, and EGF, IL6, POU5F, BIRC5, SNAIL, and EGFR were decreased compared to expression with depleted medium (noted by an asterisk in Figure 3a), suggesting that the effect of CAFs differs from the simple exhaustion of nutrients and/or production of waste metabolites. A similar experiment performed with Detroit cells showed that coculture with CAFs changed expression of another gene set (Figure 3b), suggesting that cancer cell response to CAFs differs among primary tumor cells and metastatic cells.

NANOG, a gene associated with pluripotency, was almost uniformly upregulated in FaDu cells when cocultured with media obtained from patient-derived CAFs (compared to control fresh medium) (Figure 3a, Supplementary Tables S2 and S4). Interestingly, cocultured FaDu also showed significantly downregulated expression of IL6. Nevertheless, the effect on gene expression in FaDu cells significantly differed between individual CAFs-derived conditioned media. In Detroit cells, uniform upregulation of PGE2S and downregulated expression of BIRC5 and MKI67 due to the coculture were shown.

The correlation analysis revealed that the expression of many cancer-associated genes such as PGE2S, EGFR, CAV1, NFKB, FOLR1, COX2, BCL2, VEGFA, and POU5F was closely 
related and proportional to the size of the area of tumor colonies in coculture experiments and that the area of tumor colonies was in negative correlation with CCL2 expression (see Figure 4d, Supplementary Table S3).

Regarding clinicopathological conditions, coculture of FaDu cells with CAFsconditioned media derived from patients who subsequently underwent recurrence (M.9.1 and M.7.1) significantly increased the SOX2 expression in FaDu cells compared to coculture with CAFs-conditioned media derived from patients who did not undergo recurrence. No other similar associations with recurrence and/or p16 status were observed in either FaDu or Detroit 562 cells.

a FaDu

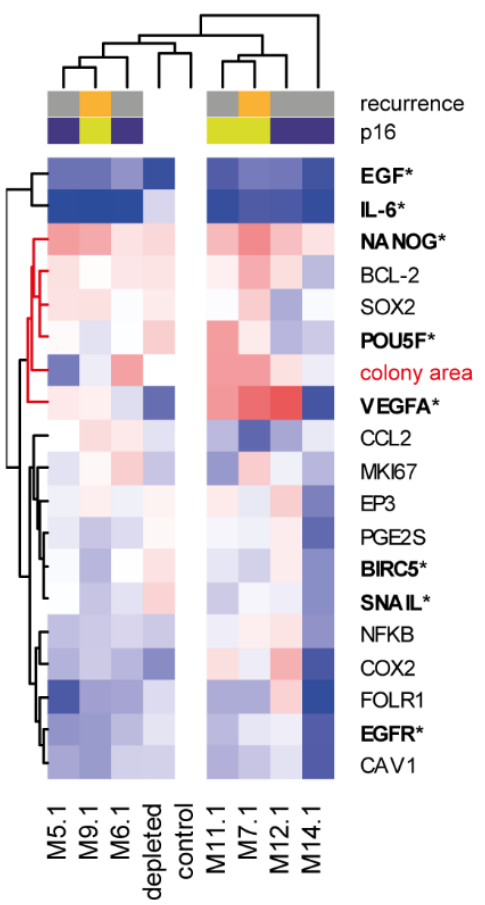

b Detroit 562

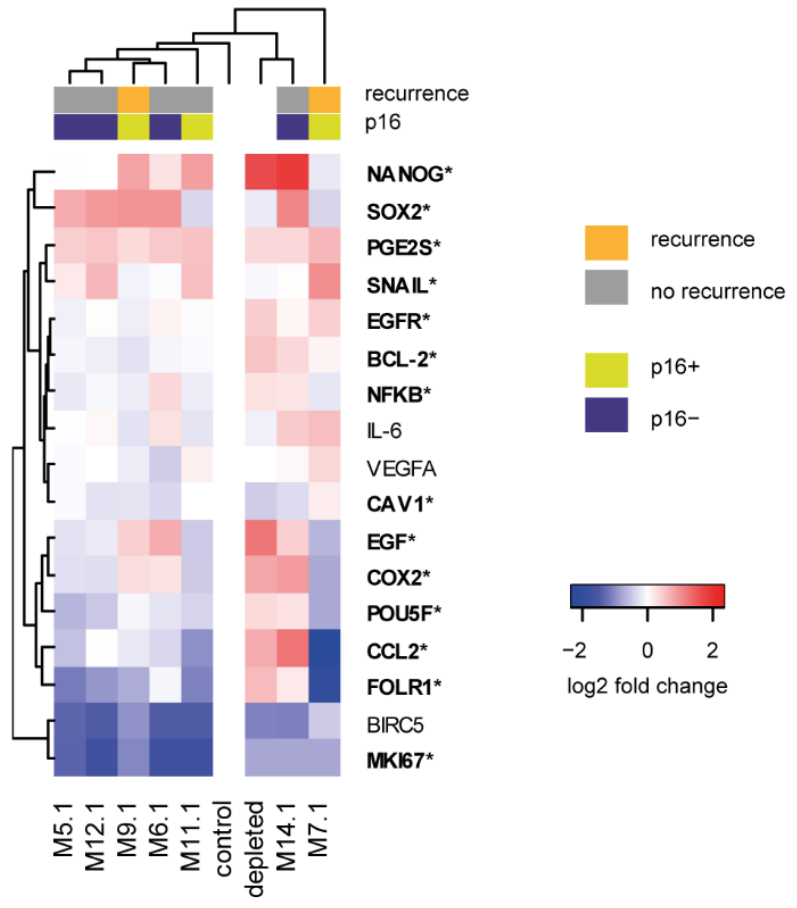

Figure 3. The effect of CAF-derived media (CMCAF) on gene expression in FaDu and Detroit cells. (a) Gene expression pattern of FaDu cells relative to non-co-cultured FaDu cells in log2 fold change, together with the log2-transformed colony area. Red cluster branch indicates genes clustered with colony area, see Figure $4 \mathrm{~d}$ for correlations. (b) Gene expression pattern of Detroit 562 cell line cocultured with patient-derived CAFs; the analogue of (a). Genes highlighted in bold with an asterisk indicate significant change compared to the $24 \mathrm{~h}$-depleted medium. Columns represent patients; rows represent genes. Gene expression levels are indicated by color: red denotes upregulation; blue denotes downregulation. 
a $\mathrm{FaDu}$

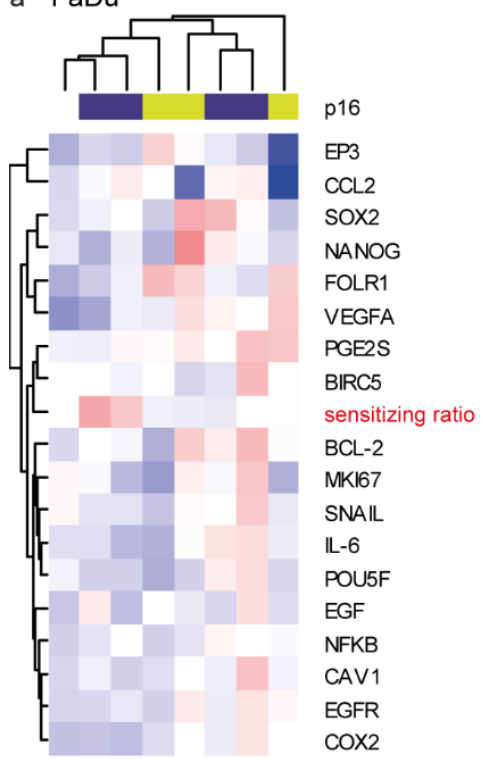

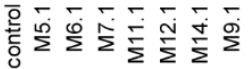

b
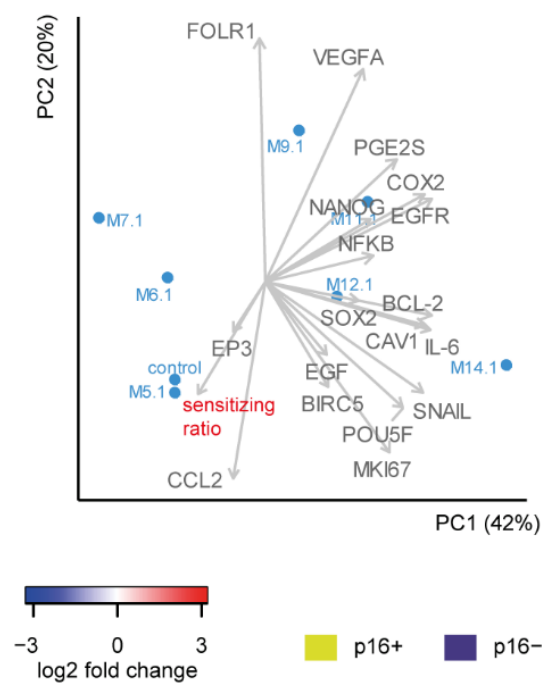

$\mathrm{p} 16+$ c Detroit 562
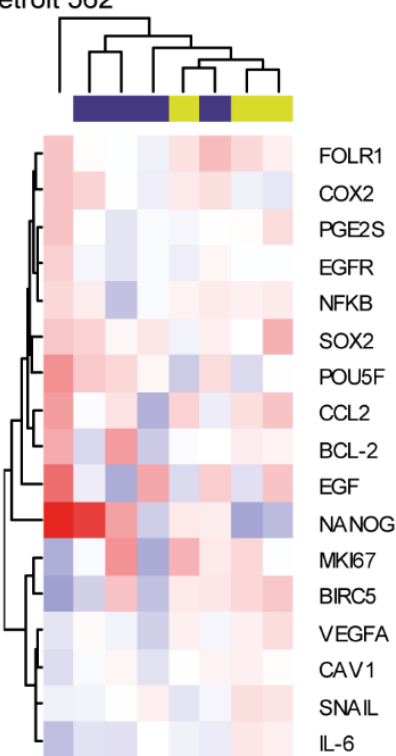

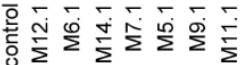
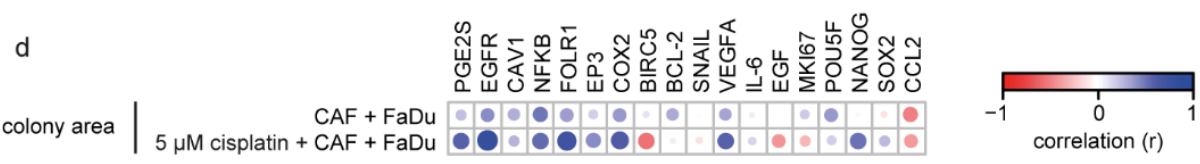

Figure 4. Effect of cisplatin on gene expression and its association with sensitizing ratio. (a) Heatmap of gene expression shown as a $\log 2$ fold change relative to individual CAF cocultured cisplatin untreated FaDu cells (to remove the among-CAF effect, the gene expression after coculture with each CAF (without cisplatin) was pairwise set as 0 ). The cisplatin effect on FaDu cell gene expression was CAF-specific and fell into two clusters: a cluster of CAFs M.5.1, M.6.1, and M.7.1 together with the non-cocultured control caused a general expression decrease and increase of cisplatin sensitivity, while M.11.1, M.12.1, and M.14.1 coculture resulted in the opposite; an increase in most of the studied genes and decreased/unchanged sensitizing ratio. (b) Principal component analysis of FaDu-cocultured cells. Colony-forming-based "sensitizing ratio", EP3, and CCL2 were clustered opposite to VEGFA, PGE2S, COX2, NANOG, and EGFR. (c) Gene expression pattern of Detroit 562 cells cocultured with CAF-derived media; the analogue of (a). (d) Correlation of colony area with the expression of target genes in FaDu cells cocultured with CAF-derived media, with or without cisplatin. A significant positive correlation is indicated by blue, negative by red. Size of the circle corresponds to significance (bigger circle corresponds to lower $p$-value).

\subsection{Cisplatin Response in FaDu Cells Is CAF-Specific}

Similarly to the previous step, gene expression changes in FaDu cells after coculture with medium from cisplatin-influenced CAFs (CMCAF_post-cisplatin) were associated with a colony-forming-based "sensitizing ratio". To remove the among-CAF effect, the gene expression after coculture with each CAF (without cisplatin) was pairwise set as 0 . The cisplatin effect on FaDu cell gene expression was CAF-specific: a cluster of CAFs M.5.1, M.6.1, and M.7.1 together the with non-cocultured control caused a general expression decrease of most genes (Figure 4a) and increase of cisplatin sensitivity, while M.11.1, M.12.1, and M.14.1coculture resulted in the opposite, an increase in most of the studied genes and cisplatin resistance-supporting effect. The expression increase of VEGFA, PGE2S, COX2, $E G F R$, and NANOG was characteristic for the increase of resistance, as the sensitizing ratio was in the opposite direction in the principal component analysis (Figure $4 \mathrm{~b}$ ). On the other hand, CCL2 expression was associated with sensitizing effect.

However, coculture of CAFs with Detroit 562 cells resulted in a different gene expression pattern, in which "sensitizing" and "resistance-supporting" CAFs were not clustered together (Figure 4c). 
Furthermore, individual genes correlated differently with the colony area of $\mathrm{FaDu}$ cells cocultured with conditioned media from cisplatin-treated and cisplatin non-treated CAFs. Conditioned media from cisplatin-treated CAFs caused a negative association of FaDu cells colony area with BIRC5 and EGF, and a positive association of colony area with NANOG expression, which was not seen in coculture with CAFs-conditioned media without cisplatin (Figure 4d).

\subsection{Platin Amount Accumulated to and Released from CAFs Relates to Aggressiveness}

For the previous gene expression analyses, the CAFs were first incubated with cisplatin (CMCAF+cisplatin), then the medium was changed and conditioned for $24 \mathrm{~h}$ (CMCAF_postcisplatin); see Figure 1c. In both media, the cisplatin content was measured and the amount of cisplatin in cells was approximated as a difference from platin added minus platin measured after 24. The platin amount released from CAFs (Figure 5a) was measured in CMCAF_post-cisplatin medium (cisplatin presented in CMCAF_post-cisplatin medium could only get there by efflux from CAFs). Significantly, higher amounts of cisplatin were found in CAFs derived from patients who subsequently underwent a recurrence $(p=0.016)$. The amount of cisplatin released from CAFs into CMCAF_post-cisplatin medium negatively correlated with the amount of cisplatin absorbed into CAFs in CMCAF+cisplatin medium (Figure $5 b$ ) and was also negatively associated with the area of the colonies.

a Cisplatin amount in CAFs
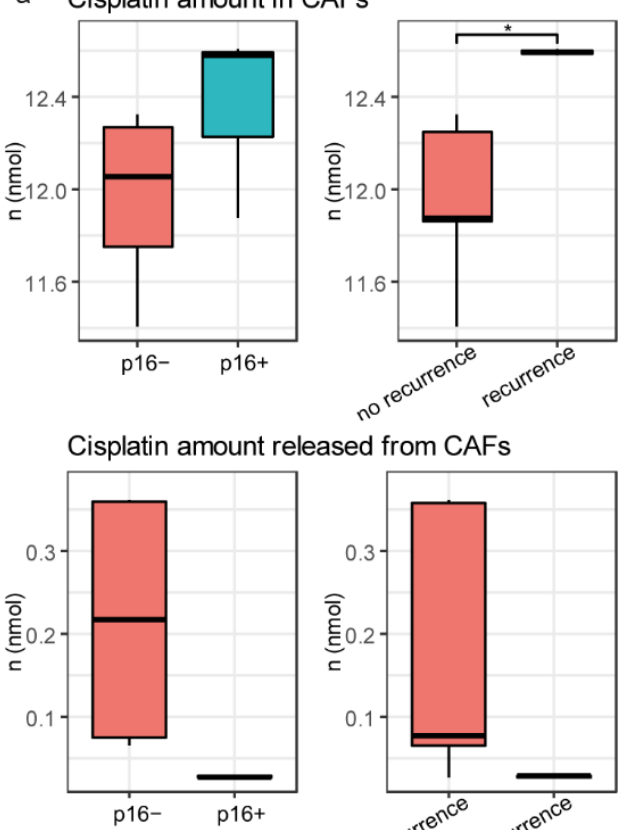

b

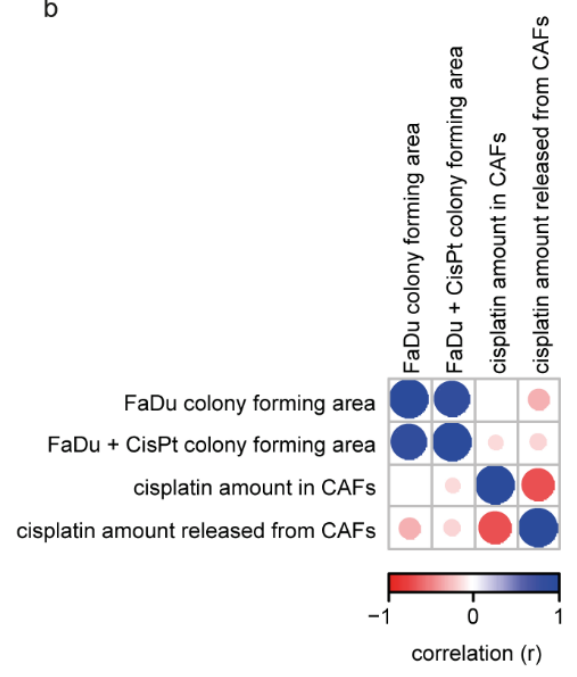

Figure 5. Cisplatin content in media and CAFs. (a) Cisplatin amount absorbed from media to CAFs is associated with patient recurrence (higher in CAFs from patients who had a recurrence). Cisplatin amount released from CAFs (CMCAF_postcisplatin medium) was not associated with p16 status and/or recurrence. (b) Correlation heatmap of colony area and cisplatin amount in CAFs and/or cisplatin released from CAFs. Asterisk indicate t-test result with $p<0.05$.

\section{Discussion}

Cancer-associated fibroblasts (CAFs) are one of the most abundant and critical components of the tumor stroma. They provide physical support for tumor cells, but also play a key role in a context-dependent promoting or delaying of cancerogenesis. CAFs can impact angiogenesis, metastasis, immunosurveillance, metabolic reprogramming, treatment resistance, stemness, and remodeling of the extracellular matrix [10]. Some of these effects need the direct contact of CAFs and cancer cells, while some involve paracrine signals [11]. 
Resistance to cisplatin is a major obstacle to improving the chemotherapy outcomes of HNSCC patients. In the present study, we found that cisplatin response in $\mathrm{FaDu}$ cells is CAF-specific as different patient-derived CAFs have a different "sensitising ratio" (Figure 2d). Expression increase of VEGFA, PGE2S, COX2, EGFR, and NANOG in cancer cells was characteristic for the increase of resistance, as the sensitizing ratio was the opposite in the principal component analysis (Figure 4b). On the other hand, CCL2 expression was associated with sensitizing effect.

We also found that patient-derived CAFs significantly affected the colony-forming capability of cancer cells by changes in expression of cancer-associated genes in tumor cells. The correlation analysis revealed that expression of many cancer-associated genes, such as PGE2S, EGFR, CAV1, NFKB, FOLR1, COX2, BCL2, VEGFA, and POU5F, was closely related and proportional to the size of the area of tumor colonies in coculture experiments. On the other hand, the area of tumor colonies was in negative correlation with CCL2 expression (see Figure 4d). Cisplatin further enhanced the positive correlation between the area of tumor colonies and gene expression of PGE2S, EGFR, FOLR1, EP3, COX2, and VEGFA in cancer cells, highlighting the importance of these genes in treatment resistance. Furthermore, cisplatin treatment of CAFs caused a negative association of colony area with BIRC5 and $E G F$, and a positive association of colony area with NANOG expression in cancer cells treated by CMCAF_post-cisplatin medium, which was not seen in CMCAF cocultured cells (Figure $4 \mathrm{~d}$ ). Taken together, coculture with CAFs can significantly enhance the expression of genes important for cancer progression. On the other hand, CCL2 expression seems to have an anti-clonogenic effect in FaDu cells. A similar effect has been observed in animal models, where CCL2 suppressed tumor growth in a T lymphocyte-independent and/or -dependent manner. $\mathrm{CHO}$ cells expressing high levels of either human or murine CCL2 formed no tumors. Sarcoma cells expressing high levels of CCL2 appeared later, grew more slowly, and were associated with an intense monocyte/macrophage infiltration [12-14]. The negative correlation of colony area with BIRC5 expression caused by cisplatin may be associated with negative regulation of autophagy by BIRC5. BIRC5 negatively modulates the protein stability of ATG7 and physically binds to the ATG12-ATG5 conjugate, preventing the formation of the ATG12-ATG5-ATG16L1 protein complex [15]. Autophagy can function as a protective cell survival mechanism against environmental and cellular stress, and perhaps causes resistance to anticancer therapies [16], as CAFs were shown to confer cisplatin resistance to tongue cancer via autophagy activation [17]. High amounts of BIRC5 could therefore be undesirable in stressed cancer cells.

Significantly higher amounts of cisplatin were found in CAFs derived from patients who subsequently experienced a recurrence. Consequently, we can speculate that higher ability to accumulate cisplatin leads to higher activation of the tumor-supporting abilities of CAFs after stress exposure. For example, interleukin- 11 was significantly up-regulated in the CAFs stimulated by cisplatin. IL-11 facilitated cancer cell chemoresistance via IL-11R/STAT3 signalling [18]. Cisplatin may also activate PAI-1 secretion from CAFs, with paracrine effects promoting esophageal squamous cell carcinoma progression and causing chemoresistance [11]. The amount of cisplatin released from CAFs negatively correlated with the amount of platin absorbed into CAFs (Figure $5 \mathrm{~b}$ ) and was also negatively associated with the area of colonies. Coculture of FaDu cells with CAFs-conditioned media derived from patients who subsequently had a recurrence significantly increased the SOX2 expression in FaDu cells. Constitutive expression of SOX2 in HNSCC cells was shown to generate a cancer stem cell-like phenotype [19].

A limitation of our study lies in the small number of studied CAF populations, and the observational and screening-based character of this study. Furthermore, our model cancer cell lines probably cannot fully reflect the whole heterogeneity of HNSCC. Nevertheless, our results showed that CAFs could promote and/or inhibit colony-forming capability and cisplatin resistance in HNSCC cells via paracrine effects, and subsequent changes in the gene expression of cancer-associated genes in cancer cells. 
Further studies should be executed to prove our findings and to disclose the exact signaling pathways enabling the cancer-promoting effect of CAFs.

\section{Materials and Methods}

\subsection{Model Cell Lines}

Detroit562 is an epithelial adherent cell line derived from the pleural effusion of metastatic pharyngeal carcinoma which contains the activating PI3K mutation. FaDu is an epithelial adherent cell line derived from hypopharyngeal carcinoma which does not carry the PI3K mutation [20]. FaDu and Detroit562 cell lines were purchased from CLS Cell Lines Service GmbH, Germany. FaDu and Detroit562 cells were cultured in MEM medium with $10 \%$ FBS, supplemented with antibiotics (penicillin $100 \mathrm{U} / \mathrm{mL}$ and streptomycin $0.1 \mathrm{mg} / \mathrm{mL}$ ) and $0.4 \mu \mathrm{g} / \mathrm{mL}$ hydrocortisone. Cells were maintained at $37^{\circ} \mathrm{C}$ in a humidified incubator with $5 \% \mathrm{CO}_{2}$ (Sanyo, Japan). The passages of cells ranged from 5 to 10 .

\subsection{Tumor Samples Collection}

The study was conducted in accord with the Helsinki Declaration of 1964, and all subsequent revisions thereof. It was approved by the ethical committee of Motol University Hospital, Prague, Czech Republic. All surgical tissue samples were obtained from HNSCC patients after they had signed informed consent. Patients with other malignancies or inflammatory and infectious diseases were excluded from this study. Patients were completely clinically examined. The tumor extent was assessed by physical examination and imaging methods (CT scan and/or MRI). Tumor samples were taken from verified HNSCC under local or general anesthesia; the inclusion criteria were as follows: (a) histologically confirmed squamous cell carcinoma with no distant metastases; (b) no previous oncologic treatment; (c) no other malignancies; (d) no inflammatory or infectious diseases. Tumor grade was evaluated following the World Health Organization (WHO) criteria, and tumor staging was determined using the TNM system (8th edition) of the International Union Against Cancer. All patients underwent surgery followed by radiotherapy or chemoradiotherapy with curative intent. Each tissue specimen underwent pathology quality control. Hematoxylin and eosin-stained sections from each sample were subjected to independent pathology review to confirm squamous cell carcinoma histological consistence. Tumor samples with $>60 \%$ tumor nuclei and $<20 \%$ necrosis were considered sufficient for the following analyses. The specimens harvested at surgery were collected in tubes containing culture medium supplemented with antibiotics and antimycotics with the addition of $1 \%$ trypsin (described below). The samples were maintained cold, and the primary cell lines were prepared within $24 \mathrm{~h}$.

\subsection{Patient-Derived Cell Cultures}

Tissue material was placed into the culture medium (MEM) with $1 \%$ added trypsin and $1 \%$ antibiotic-antimycotic solution (Santa Cruz Biotechnology, Dallas, TX, USA). The following protocol was used to process the primary culture from the sample. The tissue was first rinsed with $50 \%$ ethanol (Sigma-Aldrich, St. Louis, MO, USA) followed by phosphate buffer (Invitrogen, Waltham, MA, USA). Necrotic and adipose tissue was then removed from the tissue using a sterile scalpel, and the tumor tissue was cut into $3 \mathrm{~mm}$ pieces. Subsequently, the tumor pieces were transferred to a sterile tube with phosphate buffer and centrifuged at $4{ }^{\circ} \mathrm{C}, 2700 \mathrm{rpm}$, for $7 \mathrm{~min}$. After removing the supernatant, complete medium (MEM supplemented with 10\% FBS, 1\% antibiotic and antifungal solution, $10 \mu \mathrm{g} / \mathrm{mL}$ gentamicin sulfate and $10 \mu \mathrm{g} / \mathrm{mL}$ ciprofloxacin) was added to the sample, and the sample was cultured at $37^{\circ} \mathrm{C}$ and $5 \% \mathrm{CO}_{2}$ until the cells reached $50 \%$ confluence. After this time, the medium was changed to medium only with the addition of FBS (Biochrom, Cambridge, UK) and Pen/Strep antibiotic solution (Biochrom).

Since we were primarily interested in TME-derived fibroblasts (CAFs), selection using population overgrowing was performed. To verify the purity of the CAF cell culture, the 
cells were stained for the mesenchymal surface marker CD90 and analyzed by phasecontrast microscopy and flow cytometry (see Figure 1a,b). Cells were prepared as a single-cell suspension for FACS staining. For CD90, cells were stained with APC-CD90 antibody (Clone \#5E10, Biolegend, San Diego, CA, USA) for $30 \mathrm{~min}$ at $4{ }^{\circ} \mathrm{C}$. CD90+ and CD90- cells were identified based on isotype gating. The stained cells were acquired for analysis on a FACS Aria II flow cytometer (BD, Franklin Lakes, NJ, USA). Flow cytometry data were analyzed with FlowJo 10.7 (BD).

\subsection{Colony-Forming Assay}

FaDu cell line was cocultured with CAFs derived from the tumor tissue of HNSCC patients by using Transwell ${ }^{\circledR}$ cell culture inserts in a 1:50 ratio. For direct coculture, inserts with a $0.4 \mu \mathrm{m}$ pore size were used, which ensured that only soluble factors passed from CAFs through the insert to the FaDu cells seeded in the well below. After adhering overnight, the medium was changed for the control medium without cisplatin or medium containing $5 \mu \mathrm{M}$ cisplatin, and cells were cultivated for 3 weeks to form colonies. The concentration of cisplatin was determined based on the literature $[9,21]$. Transwell ${ }^{\circledR}$ cell culture inserts were used, which means the medium was shared between both cell populations (CAFs and cancer cells). Cisplatin was added to the cultivation medium and therefore influenced both cell populations.

After cultivation, colonies were fixed with cold methanol and visualized by trypan blue. The colonies were segmented by thresholding of the blue component of image transformed into Lab color space, where a single fixed threshold was used. Finally, the fraction of areas covered by colonies was computed.

To calculate the extent of inhibition of colony-forming capacity after cisplatin treatment of cocultured cancer cells with patient-derived CAFs, a sensitizing ratio was calculated as follows:

$$
\text { sensitizing ratio }=-\log _{2}\left(\begin{array}{l}
\frac{a_{c i s+}^{C A F}}{a_{c i s-}^{C A F}} \\
\frac{a_{c i s}^{n i s}}{a_{c i s-}^{n c}}
\end{array}\right)
$$

where $a_{c i s+}^{\mathrm{CAF}}$ and $a_{c i s-}^{\mathrm{CAF}}$ correspond to the total colony area of FaDu cells cocultured with CAFs treated or not treated with $5 \mu \mathrm{M}$ cisplatin. $a_{c i s+}^{n c}$ and $a_{c i s-}^{n c}$ correspond to noncocultured FaDu cells with and without cisplatin, respectively. Therefore, sensitizing ratio of non-cocultured cells $=0$, values $>0$ indicate a sensitizing effect of CAFs on cisplatin effect on FaDu cells, while values $<0$ indicate a resistance-supporting effect.

\subsection{Gene Expression Analysis}

Gene expression assays did not use Transwell ${ }^{\circledR}$ cell culture inserts. Conditioned media were used instead of Transwell ${ }^{\circledR}$ cell culture inserts. After the cells (CAFs) reached optimal confluence, their culture media were replaced with fresh media. After $24 \mathrm{~h}$ the conditioned media were collected (CMCAF) and replaced with media containing $5 \mu \mathrm{M}$ cisplatin [9]. After $24 \mathrm{~h}$ the media were collected (CMCAF+cisplatin) and replaced with fresh media, which were collected after an additional $24 \mathrm{~h}$ (CMCAF_post-cisplatin) (see Figure 1c). Media were frozen at $-20{ }^{\circ} \mathrm{C}$ until use in the following analyses.

FaDu cells were seeded into 6-well plates. After achieving 80\% confluency, the cells were cocultured with CAF-derived conditioned media CMCAF and CMCAF_post-cisplatin, control medium (fresh medium), or depleted medium ( $24 \mathrm{~h}$ on cancer cells without coculture). After $48 \mathrm{~h}$, cells were harvested and used for RNA isolation. Cancer cells were not in direct contact with cisplatin.

\subsection{RNA Isolation and Reverse Transcription}

TriPure Isolation Reagent (Roche, Basel, Switzerland) was used for RNA isolation. The isolated RNA was used for cDNA synthesis. RNA (1000 ng) was transcribed using a Transcriptor first-strand cDNA synthesis kit (Roche, Switzerland), which was used 
according to the manufacturer's instructions. cDNA $(20 \mu \mathrm{L})$ prepared from total RNA was diluted with RNase-free water to $100 \mu \mathrm{L}$, and the amount of $5 \mu \mathrm{L}$ was directly analyzed.

\subsection{Quantitative Real-Time Polymerase Chain Reaction}

qRT-PCR was performed using TaqMan gene expression assays with the LightCycler ${ }^{\circledR} 480$ II System (Roche, Basel, Switzerland), and the amplified cDNA was analyzed by the comparative $\mathrm{Ct}$ method using $A C T B$ and PSMB2 [22] as housekeep controls. Primer and probe sets for ACTB (assay ID: Hs99999903_m1), PSMB2 (Hs01009704_m1), NANOG (Hs04260366_g1), SOX2 (Hs01053049_s1), POU5F (Hs04260367_gH), SNAIL (Hs00195591_m1), FOLR1 (Hs01124177), MKI67 (Hs00606991_m1), NFKB1 (Hs00765730_m1), BCL2 (Hs00608023 _m1), VEGFA (Hs00900055_m1), EGF (Hs01099999_m1), EGFR (Hs01076078_m1), COX2 (Hs00153133_m1), CCL2 (Hs00907239_m1), IL6 (Hs00985639_m1), CAV1 (Hs00971716_m1), BIRC5 (Hs00153353_m1), EP3 (Hs00168755_m1), and PGE2S (Hs00228159_m1) were selected from the TaqMan gene expression assays (Life Technologies, USA). qRT-PCR was performed under the following amplification conditions: total volume $20 \mu \mathrm{L}$, initial incubation at $50{ }^{\circ} \mathrm{C} / 2 \mathrm{~min}$, followed by denaturation at $95^{\circ} \mathrm{C} / 10 \mathrm{~min}$, then 45 cycles at $95^{\circ} \mathrm{C} / 15 \mathrm{~s}$ and at $60^{\circ} \mathrm{C} / 1 \mathrm{~min}$.

\subsection{Cisplatin Concentration in CAF-Derived Media}

The concentration of platinum in media was determined by ICP-MS Agilent 7900 (Agilent Technologies, Santa Clara, CA, USA). The samples were 10 times diluted before analysis with MQ water, and the Pt concentration was measured using the isotope, 195Pt. Possible matrix effect was suppressed using an internal standard (Tl solution with a concentration of $200 \mu \mathrm{g} / \mathrm{L})$.

As the cells were treated with $5 \mu \mathrm{M}$ cisplatin, the content of cisplatin in CAFs was approximated as follows:

$$
n_{\text {cisplatin }}^{C A F}=n_{\text {cisplatin }}^{\text {added }}-n_{\text {cisplatin }}^{24 h \text { post-treatment in medium }}
$$

Cisplatin released from CAFs was measured in CMCAF_post-cisplatin medium (24 h incubation with fresh medium after cisplatin was removed). Cisplatin present in this medium could only have got there by efflux from CAFs.

\subsection{Statistical Analysis}

Principal component analysis with two components was used to show relations in multidimensional data, Pearson correlations were performed to calculate correlation coefficients between colony-forming assay and gene expression, a one-sample $t$-test was used to compare gene expression of cocultures vs. controls. R 4.0.2 [23] was used for analysis, with the following packages: ggplot2, [24], gplots 3.0.4 [25], heatmap.plus 1.3 [26], Corrplot 0.84 [27], ggpubr 0.4.0 [28], mixOmics 6.14 [29]. Unless noted otherwise, $p$-level < 0.05 was considered significant.

Supplementary Materials: The following are available online at https:/ / www.mdpi.com/1422-006 7/22/4/1912/s1.

Author Contributions: Data curation, B.P., H.H.P., D.K. and M.G.; Formal analysis, B.P., J.G., H.H.P., T.V. and M.G.; Funding acquisition, J.P., J.B. and M.M.; Investigation, M.R. and M.M.; Methodology, M.L. and T.V.; Supervision, J.P., J.B. and M.M.; Validation, J.G.; Writing—original draft, M.R.; Writingreview \& editing, J.P. and M.M. All authors have read and agreed to the published version of the manuscript.

Funding: This work was supported by funds from the Faculty of Medicine, Masaryk University to Junior researcher (Hana Holcova Polanska), by funds from Specific University Research Grant, as provided by the Ministry of Education, Youth and Sports of the Czech Republic in the year 2021 (MUNI/A/1698/2020 and MUNI/A/1246/2020) by Grant Agency of the Czech Republic 
(GACR-18-03978S), by Progres Q28/LF1 and by the Ministry of Health of the Czech Republic (NV18-08-00229).

Institutional Review Board Statement: The study was conducted in accord with the Helsinki Declaration of 1964 and all subsequent revisions thereof. It was approved by the ethical committee of Motol University Hospital, Prague, Czech Republic.

Informed Consent Statement: All surgical tissue samples were obtained from HNSCC patients after they signed informed consent.

Data Availability Statement: Gene expression, flow cytometry and colony-forming data are available as a supplement of this article.

Conflicts of Interest: The authors declare no conflict of interest.

\section{References}

1. Lemaire, F.; Millon, R.; Young, J.; Cromer, A.; Wasylyk, C.; Schultz, I.; Muller, D.; Marchal, P.; Zhao, C.; Melle, D.; et al. Differential expression profiling of head and neck squamous cell carcinoma (HNSCC). Br. J. Cancer 2003, 89, 1940-1949. [CrossRef] [PubMed]

2. Hutchinson, L. Drug therapy: Cetuximab or cisplatin in HNSCC? Nat. Rev. Clin. Oncol. 2016, 13, 66. [CrossRef]

3. Nishimura, K.; Tsuchiya, Y.; Okamoto, H.; Ijichi, K.; Gosho, M.; Fukayama, M.; Yoshikawa, K.; Ueda, H.; Bradford, C.R.; Carey, T.E.; et al. Identification of chemoresistant factors by protein expression analysis with iTRAQ for head and neck carcinoma. Br. J. Cancer 2014, 111, 799-806. [CrossRef] [PubMed]

4. Castells, M.; Thibault, B.; Delord, J.P.; Couderc, B. Implication of tumor microenvironment in chemoresistance: Tumor-associated stromal cells protect tumor cells from cell death. Int. J. Mol. Sci. 2012, 13, 9545-9571. [CrossRef] [PubMed]

5. Meads, M.B.; Gatenby, R.A.; Dalton, W.S. Environment-mediated drug resistance: A major contributor to minimal residual disease. Nat. Rev. Cancer 2009, 9, 665-674. [CrossRef]

6. Plzak, J.; Lacina, L.; Chovanec, M.; Dvorankova, B.; Szabo, P.; Cada, Z.; Smetana, K., Jr. Epithelial-stromal interaction in squamous cell epithelium-derived tumors: An important new player in the control of tumor biological properties. Anticancer Res. 2010, 30, 455-462.

7. Lacina, L.; Plzak, J.; Kodet, O.; Szabo, P.; Chovanec, M.; Dvorankova, B.; Smetana, K., Jr. Cancer microenvironment: What can we learn from the stem cell niche. Int. J. Mol. Sci. 2015, 16, 24094-24110. [CrossRef]

8. Schliekelman, M.J.; Creighton, C.J.; Baird, B.N.; Chen, Y.; Banerjee, P.; Bota-Rabassedas, N.; Ahn, Y.H.; Roybal, J.D.; Chen, F.; Zhang, Y.; et al. Thy-1(+) cancer-associated fibroblasts adversely impact lung cancer prognosis. Sci. Rep. 2017, 7, 6478. [CrossRef]

9. Gao, J.; Zhao, S.; Halstensen, T.S. Increased interleukin-6 expression is associated with poor prognosis and acquired cisplatin resistance in head and neck squamous cell carcinoma. Oncol. Rep. 2016, 35, 3265-3274. [CrossRef] [PubMed]

10. Liu, T.; Han, C.; Wang, S.; Fang, P.; Ma, Z.; Xu, L.; Yin, R. Cancer-associated fibroblasts: An emerging target of anti-cancer immunotherapy. J. Hematol. Oncol. 2019, 12, 86. [CrossRef]

11. Che, Y.; Wang, J.; Li, Y.; Lu, Z.; Huang, J.; Sun, S.; Mao, S.; Lei, Y.; Zang, R.; Sun, N.; et al. Cisplatin-activated PAI-1 secretion in the cancer-associated fibroblasts with paracrine effects promoting esophageal squamous cell carcinoma progression and causing chemoresistance. Cell Death Dis. 2018, 9, 759. [CrossRef]

12. Rollins, B.J.; Sunday, M.E. Suppression of tumor formation in vivo by expression of the JE gene in malignant cells. Mol. Cell Biol. 1991, 11, 3125-3131. [CrossRef]

13. Walter, S.; Bottazzi, B.; Govoni, D.; Colotta, F.; Mantovani, A. Macrophage infiltration and growth of sarcoma clones expressing different amounts of monocyte chemotactic protein/JE. Int. J. Cancer 1991, 49, 431-435. [CrossRef] [PubMed]

14. Conti, I.; Rollins, B.J. CCL2 (monocyte chemoattractant protein-1) and cancer. Semin. Cancer Biol. 2004, 14, 149-154. [CrossRef] [PubMed]

15. Lin, T.Y.; Chan, H.H.; Chen, S.H.; Sarvagalla, S.; Chen, P.S.; Coumar, M.S.; Cheng, S.M.; Chang, Y.C.; Lin, C.H.; Leung, E.; et al. BIRC5/Survivin is a novel ATG12-ATG5 conjugate interactor and an autophagy-induced DNA damage suppressor in human cancer and mouse embryonic fibroblast cells. Autophagy 2020, 16, 1296-1313. [CrossRef]

16. Ren, J.H.; He, W.S.; Nong, L.; Zhu, Q.Y.; Hu, K.; Zhang, R.G.; Huang, L.L.; Zhu, F.; Wu, G. Acquired cisplatin resistance in human lung adenocarcinoma cells is associated with enhanced autophagy. Cancer Biother. Radiopharm 2010, 25, 75-80. [CrossRef] [PubMed]

17. Liao, J.K.; Zhou, B.; Zhuang, X.M.; Zhuang, P.L.; Zhang, D.M.; Chen, W.L. Cancer-associated fibroblasts confer cisplatin resistance of tongue cancer via autophagy activation. Biomed. Pharmacother. 2018, 97, 1341-1348. [CrossRef] [PubMed]

18. Tao, L.; Huang, G.; Wang, R.; Pan, Y.; He, Z.; Chu, X.; Song, H.; Chen, L.-B. Cancer-associated fibroblasts treated with cisplatin facilitates chemoresistance of lung adenocarcinoma through IL-11/IL-11R/STAT3 signaling pathway. Sci. Rep. 2016, 6, 38408. [CrossRef]

19. Keysar, S.B.; Le, P.N.; Miller, B.; Jackson, B.C.; Eagles, J.R.; Nieto, C.; Kim, J.; Tang, B.; Glogowska, M.J.; Morton, J.J.; et al. Regulation of head and neck squamous cancer stem cells by PI3K and SOX2. JNCI 2016, 109, djw189. [CrossRef] 
20. Wirtz, E.D.; Hoshino, D.; Maldonado, A.T.; Tyson, D.R.; Weaver, A.M. Response of head and neck squamous cell carcinoma cells carrying PIK3CA mutations to selected targeted therapies. JAMA Otolaryngol. Head Neck Surg. 2015, 141, 543-549. [CrossRef] [PubMed]

21. Yang, Z.; Liao, J.; Carter-Cooper, B.A.; Lapidus, R.G.; Cullen, K.J.; Dan, H. Regulation of cisplatin-resistant head and neck squamous cell carcinoma by the SRC/ETS-1 signaling pathway. BMC Cancer 2019, 19, 485. [CrossRef] [PubMed]

22. Eisenberg, E.; Levanon, E.Y. Human housekeeping genes, revisited. Trends Genet. 2013, 29, 569-574. [CrossRef] [PubMed]

23. R Core Team. R: A Language and Environment for Statistical Computing; R Foundation for Statistical Computing: Vienna, Austria. Available online: https:/ / www.R-project.org/ (accessed on 9 February 2021).

24. Wickham, H. ggplot2: Elegant Graphics for Data Analysis; Springer: New York, NY, USA, 2016.

25. Warnes, G.R.; Bolker, B.; Bonebakker, L.; Gentleman, R.; Huber, W.; Liaw, A.; Lumley, T.; Maechler, M.; Magnusson, A.; Moeller, S.; et al. gplots: Various R Programming Tools for Plotting Data. R Package Version 2.17.0. Available online: https: / / www.scienceopen.com/document?vid=0e5d8e31-1fe4-492f-a3d8-8cd71b2b8ad9 (accessed on 9 February 2021).

26. Day, A. Heatmap.Plus: Heatmap with More Sensible Behavior. Available online: https:/ /CRAN.R-project.org/package=heatmap. plus (accessed on 9 February 2021).

27. Wei, T.; Simko, V. R Package "Corrplot": Visualization of a Correlation Matrix. Available online: https://github.com/taiyun/ corrplot (accessed on 9 February 2021).

28. Kassambara, A. ggpubr: 'ggplot2' Based Publication Ready Plots. Available online: https://CRAN.R-project.org/package= ggpubr (accessed on 9 February 2021).

29. Rohart, F.; Gautier, B.; Singh, A.; Le, C.K.-A. mixOmics: An R package for 'omics feature selection and multiple data integration. PLoS Comput. Biol. 2017, 13, e1005752. [CrossRef] [PubMed] 